\section{Peer Competition and Cooperation}

\author{
Ivan Dario Gonzalez-Cabrera
}

Konrad Lorenz Institute for Evolution and

Cognition Research, Klosterneuburg, Austria

\section{Synonyms}

Competition; Cooperation; Intragroup competition; Intragroup cooperation

\section{Definition}

Peer competition is a form of social interaction in which at least two organisms of a peer group strive to obtain a limited resource or achieve a certain goal. Peer cooperation is the social interaction between at least two organisms of a peer group in which at least one of them acts in a way that fosters the benefit of other group members, regardless of whether those actions are beneficial or not to the actor herself.

\section{Introduction}

The study of peer competition and cooperation is essential for providing a theoretical account of human nature and its distinctive cognitive and motivational psychology. Research in these areas also has important practical implications. An accurate understanding of peer competition and cooperation is crucial, for instance, for understanding the factors that drive human conflict, as well as for overcoming the challenges that require collaborative effort, such as building and maintaining a large-scale irrigation system for agricultural production.

Peer competition and peer cooperation can be intuitively seen as opposing phenomena. However, depending on multiple factors, they might be complementary. In a population divided into groups, for instance, members of each group may cooperate with their peers in order to compete with neighboring groups. Alternatively, they may compete with their peers as a means of choosing the best cooperative partners and demonstrate that they are reliable cooperative partners. For instance, if subjects can choose with whom they wish to interact, this may create competition to be more generous or loyal than others.

Drawing upon the developmental and comparative psychology literature, this article discusses the biological and cultural factors influencing the psychology of peer competition and peer cooperation in humans. Data from comparative psychology are useful to address questions about cognition from a broad evolutionary perspective. Developmental evidence, in turn, provides crucial information about how cognitive capacities emerge during the organism's life span, which will help to better illuminate how developmental trajectories are shaped by evolutionary processes. 
A special emphasis will be given to cooperative behavior, which, arguably, encompasses the most distinctive cognitive traits of the human species. The article ends by examining one of the most promising avenues of research on the psychology of peer cooperation: the shared intentionality hypothesis.

\section{Evolutionary and Psychological Perspectives of Peer Competition and Cooperation}

Peer competition and peer cooperation each possess a psychological and an evolutionary dimension. As such, research can focus on either the psychological mechanisms of competition and cooperation or the evolutionary explanations for them. For example, testosterone levels mediate competitive behavior when social challenges need to be confronted, and may also promote prosocial behaviors when this behavior enhances social status - e.g., when punishing norm violators brings reputational benefits to the punisher. The mechanisms involved in testosterone production are shaped by evolutionary forces that often have cascading effects on other traits. It has been argued, for example, that selection for increased social tolerance in the Middle Pleistocene led to a decrease in testosterone levels (or perhaps reduced androgen receptor densities), which resulted in changes in human craniofacial morphology. The evolutionary trajectory of the hominin lineage seems to be characterized by both an overall decrease in aggressive behavior and an increase in social tolerance (GonzalezCabrera 2017).

Much of the literature on the evolutionary dynamics of competition and cooperation focuses on formal mathematical models that apply insights from game theory. Models that focus exclusively on the ultimate explanation of cooperation (i.e., the reason why cooperation evolved), however, do not explain much about the psychological capacities or the specific neural systems that implement those cooperative behaviors. This is because cooperative behaviors could be implemented by different sets of psychological processes and neural structures that are functionally equivalent. As a result, a diverse range of mechanisms could evolve by natural selection to serve the same biological function. Many brain structures can serve the same purpose, for instance, in the same way that multiple morphological structures, such as wings in insects and birds, were selected for flying. Conversely, attention to the proximate mechanisms of cooperation (i.e., those psychological and biological substrates that explain how the organism reacts in the way that it does) helps to constrain evolutionary explanations, narrowing down the specific path by which cooperation could have emerged in a species. Thus, considerations about the proximate mechanisms of competition and cooperation are crucial for building more detailed models of the evolution of social behavior in humans and nonhuman animals.

For the most part, this article will set aside formal discussions in game theory and evolutionary game theory in order to focus on competition and cooperation primarily as psychological phenomena. This requires drawing a conceptual distinction between the evolutionary and the psychological variants of these phenomena. From a purely evolutionary point of view, competition and cooperation can be understood in terms of the fitness consequences that a certain behavior has. Competitive behaviors are those that provide a fitness benefit for the actor and which are selected for because of their beneficial effect on the actor; cooperative behaviors, in contrast, are those that provide a benefit to other individuals and which are selected for because of their beneficial effect on those individuals who receive the fitness benefit. However, an organism need not have a mind for either competition or cooperation in the evolutionary sense. Viral and bacterial pathogens, for instance, cooperate in this sense when they cause human respiratory diseases. Psychological competition and cooperation, in contrast, are understood in terms of the intention of the actor. While competitive behaviors are those with which individuals intend to gain something (e.g., by or establishing physical superiority over others), cooperative behaviors are those performed by individuals acting with the intention 
to benefit others, regardless of whether they intend to benefit themselves.

The rest of the article is organized into three sections. The first section outlines the conceptual framework of peer competition, its developmental trajectory, cultural differences, and the effect of both biological and cultural factors on those differences. The second section examines the psychological literature on peer cooperation. For theoretical purposes, the large body of empirical research on peer cooperation will be divided into two main lines of research: one focused on the mechanisms for generating the benefit of cooperation, and another focused on the mechanisms for distributing it. Generating and distributing the benefit are the two problems that individuals need to solve in order to achieve and maintain cooperation. Particular attention will be paid, to how the psychological mechanisms that deal with those problems emerge during human development and how they arose in evolutionary time. The third section focuses on the recent surge of psychological research on shared intentionality. The study of shared intentional psychology has provided a new and fruitful research program for understanding the distinctive nature of human cooperation. Competing accounts concerning the evolution and influence of shared intentionality on human cooperation will be analyzed.

\section{Peer Competition}

Understanding the phenomenon of peer competition not only requires conceptual clarity about what "competition" means but also some appreciation of the nature of peers and peer groups. Broadly construed, peers are those members of one's social group with whom one shares certain social features such as age, interests, education, and social status. Overall, peers are psychologically relevant because they are likely to influence each other's beliefs, values, and behavior. Depending on our explanatory interests, some features of social groups such as age and biological sex might be especially important in defining peer groups, while others such as spatial proximity might not. In many competitive sports, for instance, the age and biological sex of players are relevant to determining group membership, while their spatial distribution across the field is not. Careful attention to these defining features of peer groups is important because they make some patterns of social influence more salient than others and peer groups may display different forms of organization and social dynamics. For example, different forms of interaction can be observed among children of different ages, and further patterns of interpersonal relationships can be discovered when biological sex is taken into consideration.

Derivatively, peer competition can be roughly understood as the social interaction between at least two organisms within a peer group for a limited resource. Although, at a very general level, all species engage in biological competition to survive and reproduce, peer competition in humans is characterized by a set of distinctive psychological mechanisms. One salient difference is that competition in our species is significantly influenced by culture via implicit and explicit social norms. The effect of culture on competitive behavior is, of course, a function of different biological factors that can be shaped by evolutionary forces. For example, while some adaptations for peer competition emerge with age and enculturation, some others allegedly depend on biological sex. Understood as the systematic study of the biological basis of all social behavior, early sociobiology tended to overemphasize the role of biological factors and the importance of biological evolution in shaping human social behavior. However, subsequent advances in evolutionary psychology have become more conscious of this limitation by paying more attention to the role of culture and evolution-like processes of cultural change. To the extent that distinctive forms of human peer competition are largely determined by cultural factors, the emergence of these special forms of competitive behavior deserves further discussion. 


\section{Developmental Trajectory of Peer Competition}

While the relative impact of culture in human competition increases with age, adaptations for early peer competition seem to be strongly canalized in development: the emergence of those adaptations appears relatively impervious to variations in the environment or genotype of the organism. For example, human newborns have to compete for parental resources with both older and even yet-to-be-born siblings, because parents might continue to invest in their older children or invest in a new offspring as soon as the mother resumes ovulation. Thus, infants and toddlers need to appeal to others by engaging caregivers emotionally and, later in life, by monitoring their tastes and intentions using specialized cognitive mechanisms to secure adult attention (Hrdy 2009; Tomasello and Gonzalez-Cabrera 2017).

These adaptations for early competition are likely the result of concurrent processes of social selection in which conspecifics compete for access to resources other than mates. With the evolution of cooperative breeding in Homo erectus around 2-1.8 million years ago, hominin mothers could have babies at more closely spaced birth intervals than other apes due to the help received from others. Cooperative breeding is a social system in which offspring receive care not only from their parents but also from other group members, often called "alloparents." Thus, in a cooperative breeding system, mothers had to divide their care and attention to offspring more than other apes and to delegate responsibility for their offspring's care to alloparents. Children would then have had to compete more actively for care and attention, and they would have had to learn to deal with different adults more flexibly.

During early (1-6 years of age) and middle childhood (6-12 years of age), children are still engaged in a process of competition with siblings and peers for the care and attention of adults. Children often obtain attention from adults by making themselves useful to them in everyday activities. This is especially clear in naturalfertility populations in which people make no conscious effort to control fertility and, therefore, population growth depends primarily on physiological and ecological factors affecting fecundity. Children who are situated in these populations help their parents and caregivers in various ways, such as harvesting, fishing, collecting shellfish, and foraging for fruit (for a review, see Kramer 2010). In doing so, they contribute significantly to their own consumption and the economic output of the group.

Around age 6, children increasingly display what is known as "interference competition." Interference competition occurs when an individual's actions prevent another individual from achieving a goal. This is a special form of competition since it requires physical interference individuals may also compete with one another by capturing resources faster than their competitors without the need for physical interference. Peer interference competition is a highly debated topic in the literature, and this is due, in particular, to gender and cross-cultural differences in the way it is expressed. For example, it is commonly assumed that males who actively attempt to prevent others from achieving their goals may gain a reproductive advantage. In contrast, empirical evidence suggests that females engage in interference competition primarily when resources are scarce and that they avoid direct interference competition to reduce the probability of incurring physical harm through retaliation. The supposed biological rationale behind these differences, as one would expect from early sociobiological models of social behavior, is that females bear greater responsibility than males for their offspring's survival. Hence, they must avoid physical harm, including interference competition that might lead to retaliation.

This view is supported by some cross-cultural studies that indicate that female aggression is primarily directed toward other women and that physical aggression is typically used by a female when her own life or her children's lives are at risk. Since elimination of direct competitors through coalitionary support reduces the probability of harmful retaliation when the coalition outnumbers the victim, it is thought that social exclusion is a more effective strategy among females than males. Evidence from some Western 


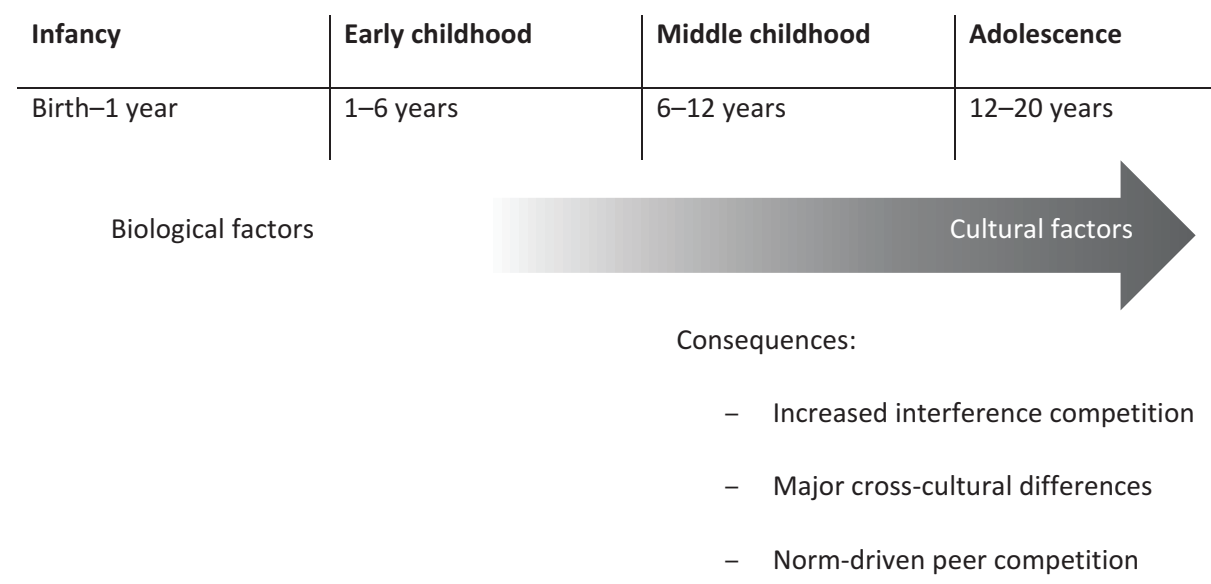

Peer Competition and Cooperation, Fig. 1 Consequences of cultural factors on peer competition through four developmental stages before adulthood

samples seems to indicate that this strategy is indeed more common among women (Benenson et al. 2013). However, gender-based differences in interference competition can be alternatively explained as a response to male-dominated environments as research on gender inequality in the workplace in industrialized Western societies seems to indicate. Further cross-cultural data is therefore required to settle this debate.

As children become more enculturated, role models and social norms begin to influence their competitive behavior (see Fig. 1). While there are few differences in children's competitive behavior during early childhood, children from different cultural backgrounds begin to differ in their competitive behavior by middle childhood. For example, equal sharing is common among peers during early childhood, especially when children collaborate to obtain a particular resource. However, as will be discussed in the section below, developmental trajectories for costly sharing diverge across cultures around middle childhood, in line with differences in the sociocultural niches that children experience (Callaghan and Corbit 2018).

\section{Cross-Cultural Differences in Peer Competition}

In general, the emergence of prosocial tendencies seems stable across developmental niches, when prosocial behavior is relatively low cost. Nonetheless, cross-cultural differences become salient under costly conditions in situations involving both helping and sharing (Blake et al. 2016). While disadvantageous inequity aversion (avoidance of receiving less than a peer) emerges by middle childhood across all populations studied, advantageous inequity aversion (avoidance of receiving more than a peer) has been shown to emerge only in some of the populations studied and only later in development. Overall, these results suggest that cross-cultural variation in adult norms of resource distribution shapes the acquisition of fairness behavior during childhood.

Adults influence children's sharing behavior, but the nature and extent of this influence varies across populations. This variation might be explained by differences in children's imitation of adult behavior in different cultural settings. Children in collectivist societies exhibit higher levels of imitation than children in individualist societies for instrumental tasks such as the retrieval of a prize from a puzzle box or a necklace-making activity (Clegg and Legare 2016). Collectivist societies typically emphasize obedience and conformity, especially children's obedience to authority figures such as parents and elders, whereas individualist societies emphasize children's autonomy and independence. Thus, it is thought that children in collectivist societies obey authority figures more than 
children in individualist societies, which results in increased imitation.

The influence of adults and cultural variation on competitive behavior often reduces potential conflict as interference competition becomes more common among children and adolescents. Cultural norms about the distribution of resources, for instance, shape social structures by regulating dominance hierarchies among peers. Dominance can be roughly understood as differential access to resources, territory, and relative power over others. Beginning in early childhood, implicit dominance hierarchies are established based on individual differences in physical prowess and social skills. But individual differences in dominance do not seem to significantly influence children's survival, as much of the necessary care and attention is already provided by parents and other adult caregivers. The importance of both dominance hierarchies and local cultural norms for the individual's fitness comes later in life.

\section{Norm-Driven Peer Competition}

As children progress into middle childhood, dominance is progressively determined by achievements in diverse cultural domains unique to humans, such as intellectual, musical, artistic, or athletic performance. Competition intensifies in adolescence as individuals begin to compete for reproductively attractive mates, which makes them increasingly aware of their place within the hierarchy. Most social interactions in children are regulated by models provided by adults or straightforward norms that decrease the cost of competition among the young, facilitating them to compete for social status. This is in stark contrast with chimpanzees who, along with the bonobo, are one of the closest living relatives of humans. Juvenile male chimpanzees do not form a dominance hierarchy with their peers (Sandel et al. 2017). One possible explanation for this is that male chimpanzees begin to establish rank by dominating adult females, but adolescent males are often not physically prepared to face female retaliatory behaviors. This is also likely the case in bonobo populations in which there is no exclusive male dominance.

Social norms can regulate peer competition in a way that suppresses dominance hierarchies to a significant degree. This is evident in huntergatherer societies, which are consistently characterized as egalitarian, in the sense that political leadership is weak and ranking and stratification among adult males is relatively absent. Egalitarian behavior is found in a wide range of ecological settings, indicating that this feature of their social organization is the deliberate result of the group members' actions (Boehm et al. 1993). This egalitarian social structure, in particular, is considered a consequence of reverse dominance hierarchies in which leaders are dominated by subordinates who disapprove of hierarchical behavior. In this way, aggressive and competitive tendencies among humans are reoriented through cultural processes in a more prosocial direction.

These reverse dominance hierarchies are typically encoded in social norms that work as leveling mechanisms that prevent inequalities of wealth, power, and prestige. Among the !Kung, for instance, hunting success is known to be quite variable, but successful hunters are denied the opportunity to use their skills to build wealth and prestige. Rather than technical limitations, it is the value system of noncompetitive, egalitarian hunter-gatherers that seems to limit the development of agriculture. In these societies, sharing is imposed, and the accumulation of personal possessions such as clothing, tools, weapons, or bead ornaments is sanctioned. Hence, these rules restrict both the progressive accumulation of wealth and the necessary investments that make agriculture possible.

Cross-cultural studies of behavior in experimental economics reveal that models based on self-interest often fail at predicting competitive behavior in small-scale societies (Henrich et al. 2005). Experimental economics uses experimental methods to study theoretical predictions of economic behavior. Research in this branch of economics has used a wide range of experimental paradigms including ultimatum, public goods, and dictator games to show that cultural, grouplevel differences such as degree of market 
integration (i.e., participation in wage labor and market exchange) and the relative importance of cooperation (i.e., the degree to which economic life depends on cooperation with non-immediate kin) significantly explain the level of prosociality expressed in these experimental games.

This cooperative behavior covaries with the costly punishment of noncooperative norm violators or, in other words, with the willingness of group members to pay the costs of punishing violators of prosocial group norms. Both evolutionary models and experimental research suggest that costly punishment is what allows norms of fairness and other prosocial behaviors to remain stable against invading defectors who behave in a selfish or antisocial manner. Yet, costly punishment is also able to stabilize arbitrary or even maladaptive norms within the group. These norms can spread in a population because, as the experimental evidence shows, children are able to imitate, for instance, the costly punishment of both equal and unequal offers in economic games from adult models, and the rates of imitation increase with age (Salali et al. 2015). That is, even when adults use costly punishment against those who make fair offers in experimental games, children imitate this behavior as if they were enforcing an antisocial norm of unfair behavior.

The above results illustrate how social norms not only curb competition as seen in societies with dominance hierarchies but can regulate competition in ways that favor increasingly hierarchical societies as well. Hierarchical organization is common in pre-state tribes headed by chiefs or "big men." Social organization in large-scale societies is also characterized by top-down power structures. Differences in social organization are fostered by variation in certain kinds of cultural practices and institutions. Storytelling, for instance, may help to broadcast social norms that coordinate prosocial behavior and promote cooperation (Smith et al. 2017). The so-called big gods and organized religion, in contrast, may foster the rise of increasingly stratified societies. Big gods are morally concerned deities who judge people's behavior and exert supernatural punishment over norm transgressors. These moralizing gods are thought to play an important role in the emergence of complex societies by fostering prosocial behaviors such as cooperation and self-sacrifice (Norenzayan 2013). However, although there is a correlation between big gods and the rise of politically complex societies, the rise of big gods seems to have only played a causal role in maintaining and promoting social inequality (Watts et al. 2016).

In hierarchical social landscapes, individuals will compete intensely to climb the social ladder, which is not the case in societies with flatter social structures. If the social structure is hierarchical, individuals will try to optimize their position in the hierarchy based on an initial set of biological and culturally inherited conditions (such as education or inherited wealth) via individual withingroup competition. This generates distinctive selective regimes that act on human populations. Egalitarian societies may have favored traits such as cooperation and low fertility, while inequality could have increased selection for within-group competition for social status, high fertility, and other selfish behaviors. Thus, individuals in the highest social classes within stratified societies may invest in cultural institutions that favor their own social status.

This investment in cultural institutions creates conditions for cultural group selection. Models of cultural selection extend the Darwinian account of evolution by natural selection in order to explain cultural change. Cultural group selection, in particular, accounts for the evolution of cultural traits as a function of the competitive advantage that these traits confer to social groups. Most evolutionary models assume that group-beneficial traits are evolutionarily unstable. They are thought to be unstable because selfish individuals who do not collaborate with the group-beneficial traits can thrive within the group. But social interactions can result in multiple stable equilibria. This is because many different forms of social organization can become stable within a group if they are sufficiently supported by social norms and if there is concurrent punishment against norm violators (Boyd and Richerson 1992). Religious beliefs about big gods, for instance, are stable within the group because they are coupled with severe social sanctions and the threat of divine punishment, 
both of which deter free riding. Under these conditions, the costs and benefits of a specific behavior are transformed by the rewards and punishments that are associated with those social norms. With groups enforcing a variety of social norms that regulate within-group competition, selection can act upon different kinds of social behavior as well as the social norms that make them internally stable within the group.

On this view, an explanation of why stratified societies evolved could implicate group-level Darwinian processes. In other words, these societies evolved as a result of variation in norms that regulate within-group competition, as well as the selective advantage that more stratified societies obtain as a result of between-group competition. Simulation modeling suggests that stratified societies are more able to survive resource shortages in variable environments by secluding mortality in the lower classes and thus keeping the population low relative to carrying capacity (Rogers et al. 2011). In both variable and constant environmental conditions, the demographic instability that is generated by unequal access to resources also results in intense migration. This migration, in turn, drives the spread of such societies and the social norms that favor unequal resource distribution, even when population size is relatively small.

Moreover, since stratified agriculturalist societies were able to grow bigger by producing more food and resources, they were also more motivated, arguably, to expand and conquer neighboring territories, creating a strong incentive to invest in organized warfare. Evidence for warfare among prehistoric hunter-gatherers exists, but this evidence becomes clearer in the archaeological record during the beginning of the agricultural revolution around 10,000 years ago, when humans transitioned from hunting and gathering to farming.

To sum up this section, the developmental trajectory of human peer cooperation is characterized by the increasing importance of culture. As children become more enculturated, cross-cultural differences in competitive behavior become more salient. These cultural differences owe primarily to social norms that regulate peer competition within the group - either by diminishing hierarchical social organization or by supporting it. The evolutionary stability of these norms depends on costly punishment, which can be transmitted early in childhood via imitation of adult models. As will be discussed in the next sections, social norms do not only regulate peer competition but depend on distinctive forms of peer cooperation for their existence. In the human lineage, the psychological mechanisms of peer competition and cooperation are highly intertwined.

\section{Peer Cooperation}

Norm-driven forms of peer competition are closely connected to human cooperative skills since they require norm compliance. The fact that people routinely comply with norms is genuinely puzzling since individuals frequently have selfish evolutionary interests in violating them. Certainly, norms can be enforced by costly forms of punishment. However, costly punishment is paradoxical precisely because it is an extreme form of cooperation that requires the agent to refrain from incentives to avoid carrying out punishment.

Peer cooperation can be roughly understood as a type of social interaction in which at least one individual acts with the intention to foster the benefit of other group members, regardless of whether those actions benefit the actors themselves. When individuals cooperate without regard to their own self-interest, their behavior is considered a case of psychological altruism. The form of costly punishment discussed in the previous section, for instance, is often considered psychologically altruistic. However, not all cooperative behavior is altruistic in this sense. Mutualistic cooperation can be motivated by prudential reasons: an individual can cooperate with another because of the foreseen immediate or delayed returns of their cooperative behavior.

Understanding how cooperation evolves is not only a central issue in evolutionary biology but also in the psychological sciences. Various accounts of those cognitive abilities that are thought to be unique to humans often link the 
evolution of those features to the evolutionary history of cooperation in our lineage. From collective hunting to cooperative breeding, cooperation is a defining feature of human social life. For example, collaborative hunting, as observed among hunter-gatherers, seems to require the mastery of a set of special cooperative skills that go beyond those we see in our closest evolutionary relatives, the chimpanzee and the bonobo. This is because collaborative hunting relies on cognitive capacities such as role and task division, joint plans, and shared commitment, which are, arguably, uniquely human. To distinguish this psychologically rich form of cooperation from its less sophisticated counterparts, it will hereon be referred to as "collaboration."

\section{The Two Problems of Peer Cooperation}

Explanations of the evolution of cooperation have primarily focused on what is sometimes called "the problem of distributing the benefit of cooperation"- namely, how cooperation can be maintained in the face of free riders that gradually undermine cooperative enterprises. This problem arises because cooperation requires groups to distribute the benefit of these ventures in ways that incentivize individuals to cooperate over time. But, as a rule of thumb, individuals have a strong incentive to free ride - i.e., to obtain the benefits of cooperation without paying the costs of generating it. As a result, free riding individuals become more successful than cooperators who bear the cost of cooperative activities, causing cooperation to progressively decline in the population. However, explaining the evolution of cooperation demands not only an explanation of how the benefit is distributed but also of how it is generated. Successful cooperation sometimes requires skills for communication, division of labor, or task commitment that explain how the benefit is generated in a cooperative interaction. This problem is called "the problem of generating the benefit of cooperation" (Calcott 2008).

These two problems can be illustrated by a two-person "stag hunt" game, which involves a situation of social coordination. In this game, two
Peer Competition and Cooperation, Table 1 Payoff matrix of a generic two-person stag hunt game, where $a>b \geq c>d$. The payoff defined in the top-right corner cell describes a situation in which Player 1 decides to hunt a stag and Player 2 decides to hunt a hare. In this scenario, Player 1 obtains a payoff of c, and Player 2 obtains a payoff of $b$

\begin{tabular}{l|l|l|l}
\hline & \multicolumn{3}{|c}{ Player 1 } \\
\hline \multirow{2}{*}{ Player 2 } & & Stag & Hare \\
& Stag & $a, a$ & $c, b$ \\
\cline { 2 - 4 } & Hare & $b, c$ & $d, d$ \\
\hline
\end{tabular}

hunters (or players) must decide simultaneously whether to hunt for stag or hare. Each player can successfully hunt a hare alone, which makes hare hunting the safer option. Hunting a stag, in contrast, requires cooperation from both players. If the two hunters agree to cooperate, they can successfully catch a stag, which is the preferred and more valuable game. If both players succeed in hunting a stag, the game is shared equally, as described in the payoff matrix in Table 1. This matrix defines, then, a solution to the problem of distributing the benefit of cooperative hunting: equal sharing. However, choosing to hunt a stag is risky since the other player may choose not to cooperate. If that is the case, the hunter who decides to go after the stag ends up empty-handed since the successful hunting of a stag requires both players. Thus, in order to generate the benefit of cooperative hunting, the two players need to coordinate their choices and to trust each other in their commitment to hunting a stag.

This distinction is psychologically relevant since mechanisms dealing with these two problems might be supported by two distinct sets of psychological mechanisms that are dissociated in both development (ontogeny) and evolution (phylogeny) (for a review, see Warneken 2018). On this view, while the capacity to generate the benefit of cooperation has evolutionary roots that we share, for the most part, with other great apes, there is little evidence that our ape relatives possess similar skills for distributing this benefit. This set of skills, furthermore, has a distinctive developmental onset. While many key skills for generating benefit through cooperation arise early in 
Peer Competition and Cooperation, Table 2 Time of onset of children's skills for generating and distributing the benefit (see Warneken 2018)

\begin{tabular}{l|l|l}
\hline Time of onset & $\begin{array}{l}\text { Skills for } \\
\text { generating the } \\
\text { benefit }\end{array}$ & $\begin{array}{l}\text { Skills for distributing } \\
\text { the benefit }\end{array}$ \\
\hline 18 months & - Helping & \\
\hline 18 months & - Costly sharing & \\
\hline $14-18$ months & - Collaborating & \\
\hline 21 months & & - Partner choice \\
\hline 3 years & & $\begin{array}{l}\text { Disadvantageous } \\
\text { inequity aversion }\end{array}$ \\
\hline 3.5 years & & - Partner fidelity \\
\hline 5 years & & $\begin{array}{l}- \text { Reputation } \\
\text { management }\end{array}$ \\
\hline $7-8$ years & & $\begin{array}{l}\text { - Advantageous } \\
\text { inequity aversion }\end{array}$ \\
\hline
\end{tabular}

human development, the mechanisms responsible for distributing it in ways that protect cooperation against defection are acquired later, for they strongly depend on children's acquisition of local social norms. For example, while basic capacities for helping, costly sharing, and collaborating arise relatively early during the first 2 years of age, mechanisms that help to distribute the benefit of cooperative activities - such as partner choice, partner fidelity, inequity aversion, and reputation management - begin to emerge at 3 years of age or later during middle childhood (see Table 2).

\section{The Ontogeny of the Mechanisms for Generating and Distributing the Benefit of Peer Cooperation}

There is significant evidence for the partial disassociation in ontogeny of the psychological mechanisms responsible for generating and distributing the benefit of cooperation. Young children are not only able to identify cooperative partners but also seem to prefer them over uncooperative ones, as measured by their reaching behavior and preferential looking. By 2 years of age, children already possess context-sensitive expectations about equal resource distribution. At this age, for instance, children expect an experimenter to give a reward to each of two individuals who have worked together to complete a task, but not when only one of them has done all the work. It seems, however, that children's evaluations are only able to regulate their social interactions in the cooperative direction later on in development. For example, 17- and 22-month-olds tend to help both antisocial and prosocial adult partners despite their preference for prosocial individuals, and 3-year-olds share indiscriminately in experimental tasks involving distribution of resources.

The best example of the delayed emergence of mechanisms for distributing the benefit of cooperation is children's development of inequity aversion. Although children seem to expect an equal distribution of resources at a very young age, aversion to unexpected unequal distributions emerges significantly later. In fact, the two forms of inequity aversion discussed in the section on Cross-Cultural Differences in Peer Competition follow different developmental trajectories. While disadvantageous inequity aversion develops around 4 years, advantageous inequity aversion begins to emerge at around 8 years of age (McAuliffe et al. 2013).

\section{The Phylogeny of the Mechanisms for Generating and Distributing the Benefit of Peer Cooperation}

While chimpanzees and bonobos have basic capacities for generating the benefit of cooperation (such as skills for helping, sharing, and cooperating in mutualistic ways), they lack the distinctive machinery for solving the problem of distributing the benefit of cooperation. First, when a human or conspecific fails to retrieve an object that is out of reach, chimpanzees can engage in instrumental helping and even select the correct tool from a set of options. Second, although only bonobos seem to be able to engage in active forms of food sharing, chimpanzees are also capable of sharing food with conspecifics in a passive way. They give food to others, for instance, when the food is difficult to monopolize or when they experience harassment from a beggar. Third, several studies with both species of African apes, 
chimpanzees and bonobos, indicate that they can cooperate with other conspecifics in a mutualistic fashion. For example, some studies have shown that chimpanzees will wait for a partner in a cooperative task, when the partner is necessary for solving the task and, therefore, for obtaining the food reward. They even actively solicit help from them or help them (e.g., by opening the door of the enclosure), so they can cooperate in the task.

Perhaps the most salient, but controversial, examples of the phylogenetic disassociation of the mechanisms for distributing the benefit between humans and other apes come from studies on partner choice and inequity aversion. While human children prefer helpers over mean partners, great apes prefer dominant individuals as these social relationships determine access to mating opportunities, food, and other resources. Even bonobos prefer individuals that hinder others over those that help. Yet, some researchers have argued that primates possess a sense of fairness. Their reason for doing so is that primates react aversely to experimenters when they see a conspecific receive better food than themselves (Brosnan and de Waal 2003). However, evidence for a sense of fairness in nonhuman animals have been somewhat difficult to replicate under more controlled conditions (Sheskin et al. 2014), leaving the phenomenon open to alternative explanations. A different account of these aversive reactions, for instance, is that seeing another individual receive high-quality food generates the expectation in the subject of receiving the same type of food. In fact, subsequent experiments in chimpanzees suggest that these reactions are better characterized as frustration for not getting the expected food, rather than aversive reactions based on a sense of fairness or some sort of social comparison with what conspecific partners obtain.

To summarize this section, explanations of peer cooperation require an account of how the benefit of cooperative ventures is distributed among group members, so that free riding does not undermine cooperation over time. Moreover, it needs an explanation of how the benefit of cooperation is generated. Psychological evidence indicates that humans possess specialized mechanisms for addressing these two problems. While basic capacities for generating the benefit of cooperation through helping, sharing, and collaborating arise relatively early in development, mechanisms for resolving the problem of distributing the benefit of cooperation - such as partner choice, partner fidelity, inequity aversion, and reputation management - only begin to emerge later during middle childhood.

\section{Peer Cooperation and Shared Intentionality}

Even though great apes and humans seem to share many of the skills required for generating the benefit of cooperation, there are still important differences in how humans and other apes address this problem. A significant body of research in developmental and comparative psychology shows that human cooperation is characterized by a set of unique skills that fall under the umbrella concept of shared intentionality. Shared intentionality is the capacity to coordinate social interactions through the sharing of mental states such as goals and beliefs. In the stag hunt game discussed in the previous section, for instance, both players could solve the problem of generating the benefit of cooperation by sharing their intention to create a committed partnership to hunt a stag.

Cooperative skills for shared intentionality seem to be in conflict with the idea that the mechanisms dealing with the two problems of peer cooperation explained in the previous section can be easily dissociated in both ontogeny and phylogeny. Indeed, skills of shared intentionality begin to emerge early in the context of child-adult interactions, but they continue to develop well into adulthood in the context of peer interactions. One notable example is collaborative hunting in hunter-gatherers, which relies on joint goals and plans, task and role division, and shared commitment that begin to emerge during childhood. Moreover, skills of shared intentionality seem unique within the ape lineage, for comparative research has systematically shown that nonhuman great apes, with whom we share a common ancestor around 7 mya, systematically fail tests of 
shared intentionality (Call 2009). Thus, these skills are hypothesized to be adaptations for generating the benefit of cooperation that emerged, due to selective pressures unique to the hominin lineage, from an ape-like common ancestor who lacked these capacities.

Shared intentionality explains many of the features that make human cooperation different from great ape cooperation (Tomasello and Carpenter 2007). The shared intentionality hypothesis states that the distinctive nature of human cooperation is due to behavioral differences produced by the presence of these abilities in the human lineage. Different social and cooperative skills that were likely present in our ape ancestors seem to have been transformed by shared intentionality (see Table 3). First, great apes understand what other individuals see, but they simply follow another's gaze to see if they are looking at something interesting, e.g., a piece of food. If they see nothing rewarding or valuable to them, they quickly stop looking. Human children are also able to see what others see, but they are also capable of sharing the attentional state in a way that involves mutual awareness that the experience is shared, as well as a shared interest in the object or event. From a very young age, for instance, children are able to gaze at adults, point to an object, and then return their gaze back to the person with whom they are interacting. Children tend to focus their attention on objects and events that others are attending to because they seem to find it intrinsically rewarding to share those experiences with others. This facilitates the cooperative exchange of

Peer Competition and Cooperation, Table 3 Basic social and cooperative skills in great apes that are transformed by human-shared intentionality (see Tomasello and Carpenter 2007)

\begin{tabular}{l|l}
\hline $\begin{array}{l}\text { Individualistic } \\
\text { (great apes: chimpanzees } \\
\text { and bonobos) }\end{array}$ & $\begin{array}{l}\text { Collaborative } \\
\text { (humans: } 1 \text {-year-olds } \\
\text { and infants) }\end{array}$ \\
\hline - Gaze following & - Shared attention \\
\hline - Social manipulation & $\begin{array}{l}\text { - Cooperative } \\
\text { communication }\end{array}$ \\
\hline - Group activity & - Collaboration \\
\hline - Social learning & - Instructed learning \\
\hline
\end{tabular}

information. For example, children tend to show adults objects that are interesting to them and, in turn, follow an adult's gaze to experience what is interesting for them. Adults can similarly bring objects in their surroundings to an infant's attention solely by using eye gaze. This creates a psychological common ground that enables both cooperative communication and collaborative activities with joint goals.

Second, nonhuman apes use gestures and signals only to get what they want from one another, while young children also use gestural communication for cooperative purposes. Nine-month-old human infants often direct other people's attention to objects through gestures aimed to initiate joint attentional interactions. Twelve-month-old infants use pointing to share interest and attention with adults as well as to inform others of things they might not have seen or know when there is no benefit for themselves (Liszkowski et al. 2006).

Third, while nonhuman great apes regularly engage in group activities such as hunting, these activities do not seem to involve individuals cooperating through shared goals to which they are all both committed and mutually aware of one another's commitment. Group hunting may be cooperative, but not necessarily "collaborative" in the psychological sense specified at the beginning of the previous section. Experimental evidence suggests that 18- and 24-month-old children understand group activities around shared goals as involving commitment from all the parties involved. At this age, infants actively encourage an unresponsive adult to rejoin a game by communicating with them in various ways. In contrast, in the same experimental situation, chimpanzees never try to reengage their partners but rather focus on solving the task by themselves (Warneken et al. 2006).

Fourth, while social learning in nonhuman great apes is mostly opportunistic (since individuals gather information from others unilaterally, copying only the actions that are relevant to achieve the desired result), human infants focus on causally irrelevant actions that they think are intentionally demonstrated by an adult. In other words, children imitate adult models in the sense that they learn the detailed actions that others 
perform (in a seemingly intentional way) to generate a certain outcome. If an adult demonstrator knocks a music box three times before making it work, children will tend to do the same, even if they know the action is instrumentally irrelevant for making the box play the music. Opportunistic learning in nonhuman apes, in contrast, biases learning toward the emulation of causally relevant actions. Moreover, as in the music box example, adults often demonstrate to children what they should do, which makes learning a cooperative activity. When adults provide communicative cues that they are demonstrating something (e.g., by saying "This is the way we do it" or "This is the way it works"), 14-month-old infants copy the specific actions adults use much more often than when they do not explicitly instruct. If adults do not provide those cues, young children tend to only copy the result the adult achieved (Gergely and Csibra 2006). Remarkably, older children around the age of 2 not only learn imitatively, but they also seem to understand group activities with peers as normatively grounded (Rakoczy and Schmidt 2013). For example, when they learn from a model that a certain activity is performed in a certain way, children enforce the conventional rules of the activity when others violate them.

\section{The Cooperative Origins of Shared Intentionality}

Traditionally, the evolutionary origins of shared intentionality have been linked to obligate cooperative foraging, especially collaborative hunting. According to the interdependence hypothesis, our early ancestors had none of the adaptations for vertical climbing, forelimb suspension, and knuckle-walking. This precluded them from seeking protection by quickly climbing to the trees or engaging in the type of hunting that we sometimes see in chimpanzees. Given the local predatory fauna, foraging alone (seeds, tubers, or meat) would have been a very dangerous task. Foraging and scavenging in a group for safety then became necessary during the Middle Pleistocene around 780 kya. In order to face those challenges, hominins required new adaptations for collaborative foraging. When nonhuman great apes face situations in which they need to decide whether to forage alone or cooperate with others to obtain a food reward, they lack the skills of coordination and communication that facilitate the reliable coordination of action, especially in the context of risky coordination problems such as the aforementioned stag hunt game (see Table 1). Thus, on this view, hominin ancestors likely lacked these adaptations until the selective pressures for obligate cooperative foraging came into effect, creating the conditions for the gradual evolution of more planned and coordinated foraging in the human lineage.

One trouble with this traditional evolutionary account of shared intentionality is that it does not explain why many of these capacities emerge so early in ontogeny. According to an alternative account, the cooperative breeding hypothesis, the key selective pressures responsible for the development of shared intentionality were linked to cooperative breeding, which likely evolved in $H$. erectus around 1.9 mya. This new rearing environment allowed humans to wean infants relatively early and, in doing so, reduce the time periods between pregnancies. This situation, as explained earlier in the section on the Developmental Trajectory of Peer Competition, increased competition between siblings and peers who must monitor the whereabouts and intentions of adults in order to secure their care and attention (Hrdy 2009).

Consistent with this scenario, it has been argued that cooperative breeding could have selected for skills of shared attention in infants. Shared attention is not simply parallel attention. Operationally, an infant is sharing attention with her caregiver when (i) the focus of the infant and caregiver's attention is directed to the same object, (ii) the infant is able to track the caregiver's focus of attention, and (iii) the infant engages caregivers just for the sake of sharing with them that attentional state. Unlike human infants, nonhuman great apes do not seem able to share attention or other experiences with members of their own species. Chimpanzees and bonobos are indeed able to meet a version of condition (i) by focusing their attention in parallel to the same object. They can 
even meet similar requirements to those stated in condition (ii) by tracking other individuals' attentional states in competitive contexts such as food seeking. However, since these species seem to be motivated to follow another's individual gaze only for instrumental and competitive purposes, they crucially fail to satisfy condition (iii). Thus, it has been claimed that cooperative breeding favored the emergence of shared attention in our lineage because gestures and eye contact would be crucial to direct adults' attention to objects, to events, and to themselves, just for the sake of sharing with them pleasurable experiences that foster attachment and care.

Similarly, basic forms of cooperative communication could have also been a consequence of the early emergence of these shared intentional skills, for they could have emerged prelinguistically in the form of pointing and pantomiming. Nonhuman great apes sometimes point for humans in an imperative way, e.g., when they want an out-of-reach object. In contrast, human infants across different cultures point declaratively from around their first birthday. At that crucial developmental stage, human infants become highly motivated to share their interest in different objects and events by offering, showing, and pointing declaratively to them.

Overall, all these uniquely human infant behaviors would have evolved in the context of sibling and peer competition. For in the context of cooperative breeding, infants compete with each other to provide adults with positive affective feedback in order to secure care and attention. As a result, they could have used these skills to reward adults by sharing emotions, interest, and attention with them. Shared intentionality would have begun as an ontogenetic adaptation, i.e., as part of a sequence of specialized changes that enabled infants to survive the cooperative breeding environment created by their parents.

However, as it will be explained in the next section, it is also likely that these early mechanisms of shared attention and basic cooperative communication coevolved as mechanisms to facilitate social learning (and perhaps even teaching), when adult activities required skills that demanded prolonged periods of preparation and maturation. This view explains the developmental trajectory of human skills for peer cooperation as the result of selective pressures for both cooperative breeding and cooperative foraging. These selective pressures are thought to collapse into each other during middle childhood - a developmental period in which children's dependence extends from their parents and other caregivers to cooperative peers. Thus, although some capacities for shared intentionality emerge early in ontogeny, others appear relatively late to support the generation of benefits via peer cooperation.

\section{The Role of Ontogeny in the Evolution of Human Peer Cooperation}

Accounts of the evolution of human cooperation have started to pay increasing attention to theoretical work in evolutionary developmental biology or "evo-devo" for short. Evo-devo focuses on how developmental processes evolve. A key tenet of this research program is that many evolutionary processes result from changes in developmental timing. Building upon this program, a recent hypothesis about the role of ontogeny in the evolution of human cooperation considers the tradeoffs that emerge during the different stages of human development in order to provide an evolutionary account of the ontogeny of human cooperative capacities (Tomasello and GonzalezCabrera 2017). An advantage of this account is that it not only explains the evolutionary emergence of those traits but also their distinctive developmental trajectory.

The evo-devo hypothesis of the evolution of human cooperation integrates insights from both the interdependence hypothesis and the cooperative breeding hypothesis. As in the cooperative breeding hypothesis, it maintains that shared intentional capacities were initially geared to dyadic interactions between infants and caregivers. As children grow older, the model similarly assumes that most of the attention of adults is redirected toward younger individuals, which makes engaging caregivers progressively more complex. Yet, as time progresses, children also become more physically capable of helping and 
collaborating. Learning how to make themselves useful to adults in their everyday activities would have been a possible way to gain such care and attention. This is a plausible assumption given how significant children's contribution is for the overall economy of extant natural-fertility populations. By the age of 5, for instance, Hadza children provide $50 \%$ of their own caloric intake. Among subsistence agriculturalists, Maya boys provide $50 \%$ of what they consume, while girls produce the same percentage by the age of 6 , once subsistence work includes food processing and household tasks (see Kramer 2010). As a result, basic abilities for shared intentionality and collaboration in early human children could have subsequently extended into adjacent developmental periods. This is an expected result of the selective advantages these skills provide in adulthood, at the cost of little or no disruption in the overall cognitive development of the organism. For example, basic skills for shared attention, cooperative communication, helping, and collaboration would have presumably conferred a great selective advantage to hunting partners in a context in which cooperative foraging was necessary for subsistence.

Moreover, building upon the interdependence hypothesis, the evo-devo model of human cooperation assumes that selective pressures stemming from increased interdependence created demands for more complex adaptations for coordination, communication, and shared commitment. These adaptations progressively moved "upstream" into childhood as a preparation for adult activities, in the sense that they began to emerge earlier in development. Most of this preparation would have occurred during middle childhood as it is in this developmental period that children need to learn how to make decisions with others with little or no adult supervision. They are typically lowrisk decisions such as choosing those activities that interest them and match their level of competence. But these decisions must be fair and impartial as participation is voluntary rather than forced. Children must interiorize the social benefits of sharing resources with others and learn how to be reliable cooperative partners. Thus, the transition to middle childhood opened a window of opportunity to learn key cooperative skills in low-risk contexts where failure to cooperate was not lethal.

A prime example of this is children's early skills for reputation management and normatively grounded cooperation. Reputation management is the capacity to adjust one's behavior in order to be judged by others in a more positive light. Normatively grounded cooperation refers to one's capacity to understand cooperative activities as involving commitment and to enforce those commitments. These skills could have initially emerged in adult individuals as adaptations for cooperative foraging. Later in evolution, they could have begun to emerge progressively earlier in development. This is because longer periods of maturation for these skills would have given individuals an evolutionary advantage when collaborative foraging was necessary for human subsistence. The primary selective pressures would have proceeded mainly upstream from adulthood and adolescence to childhood, enhancing their sensitivity to shared commitment and to the reputational effects of their own cooperative behavior. Arguably, this capacity did not evolve in the context of child-adult interactions since these relationships are hierarchically structured and, therefore, much more sensitive to other factors such as authority and fear of punishment. In contrast, starting from adolescence, individuals must collaborate with others for basic subsistence. Individuals with enough time to develop complex skills of shared intentionality, such as a capacity to form shared commitments and enforce them normatively, would gain a selective advantage. Moreover, since the networks of allies and friends that children build during this period will endure into adulthood, investing in a progressively earlier onset of complex skills for peer collaboration would have made a nontrivial difference in their fitness.

Consistent with this hypothesis, human child development is longer when compared with any other great ape. It also includes the appearance of middle childhood as a developmental period between infancy and puberty that is distinct from the juvenile stage of other great apes (Thompson and Nelson 2011). Traces of this process can be 
observed in the increasing awareness of the child about their own social reputation and the norms that regulate their social environment. Unlike nonhuman great apes, human children seem to be concerned about others' evaluations of their cooperative and prosocial tendencies because they appear to adjust their behavior based on their prediction of how others will assess this behavior. Experimental results indicate that 5-year-old human children, but not chimpanzees, share more and steal less when they are being watched by a peer than when they are alone (Engelmann et al. 2012). Between 6 and 7 years of age, children begin to enact fairness norms against selfish individuals and exhibit a deeper understanding of the normativity of social rules as arising from social agreement and commitment (McAuliffe et al. 2017).

To conclude this section, a number of alternative hypotheses have been advanced to explain the evolution of human cooperative skills of shared intentionality. The interdependence hypothesis argues that cooperative skills of shared intentionality evolved due to the selective pressures of cooperative foraging in the hominin lineage, while the cooperative breeding hypothesis explains its emergence as a result of the selective demands of cooperative breeding. An alternative approach, grounded in theoretical insights from evolutionary developmental biology, explains the emergence of cooperative abilities for shared intentionality as a consequence of adaptive changes in developmental timing stemming from both selective pressures. Further research on the evolutionary basis of human cooperation should focus on refining and testing these alternative hypotheses.

\section{Conclusion}

Human peer competition is determined by several biological factors (e.g., age, gender, and physiology) and cultural factors (e.g., social norms). The degree to which each of these factors drives human social behavior, however, is a matter of dispute. Arguably, the influence of cultural factors increases with age as children become increasingly aware of the customs, norms, and values of their groups. Peer competition starts early in development as children must strive for adult attention due to human rearing environments of cooperative breeding that are unique among other great apes. As time progresses, competition becomes increasingly regulated by norms that influence social behavior, including sharing and distributing resources. These norms likely explain cultural differences in peer competition. Some evolutionary models might, as illustrated by some accounts of the correlation between big gods and the rise of politically complex societies, overstate the role of social norms in thwarting withingroup peer competition. However, social norms can also intensify resource and status competition by stabilizing hierarchical social organization through different forms of normatively driven punishment.

Competition and cooperation among peers are, as we have seen, interconnected phenomena. When human peers compete over resources, they often do so by cooperating with others. Similarly, norm-driven forms of peer competition are closely connected to human cooperation because they require norm compliance. From a theoretical point of view, cooperating with peers involves psychological mechanisms for generating and distributing the benefit of cooperative ventures. Extensive empirical research suggests that several psychological mechanisms that allow humans to deal with the problem of generating the benefit are shared with other nonhuman great apes. Mechanisms for distributing the benefit in humans are, in contrast, more distinctive: specifically, they are closely connected with norms that regulate within-group competition. Somewhat more controversially, some evolutionary-minded psychologists have recently argued that the distinctive trajectory of these two classes of mechanisms is evident in ontogeny as well as phylogeny. Controversy about this distinction in phylogeny comes mainly from the literature on fairness and inequity aversion in nonhuman animals. The fact that capacities for shared intentionality are uniquely human, and that some of them, at least, begin to emerge relatively late in development, also provides grounds for skepticism concerning 
the notion of a distinctive ontogenetic and phylogenetic trajectory.

Humans are characterized by psychological mechanisms of shared intentionality that have helped to coordinate increasingly complex cooperative activities in the hominin lineage. In this sense, shared intentionality can be understood as a uniquely human capacity for generating the benefit of cooperation. Shared intentionality transforms several social skills that humans share with great apes such as chimpanzees and bonobos. Three basic accounts of the evolution of shared intentionality have been proposed in the literature: the interdependence hypothesis, the cooperative breeding hypothesis, and the evo-devo hypothesis. The first account explains the evolution of cooperative skills of shared intentionality as result of selection for collaborative foraging in adults. The second argues that these skills evolved due to the selective demands of cooperative breeding. The third approach explains the emergence of these abilities through a composite explanation: selective pressures for cooperative breeding in humans, in addition to changes in developmental timing in adult skills for collaborative foraging that required longer periods of maturation, jointly explain the evolution of cooperative skills. Evidence for this hypothesis can be found in the distinctive developmental pattern of middle childhood in humans. Choosing between these competing hypotheses, however, requires further investigation.

\section{Cross-References}

Competition Between Groups
Competition For Resources
Cooperation
- Cooperative Breeding
Cooperative Foraging
Difference In Competition (Resources) Com-
pared To Asia/Europe
Dominance Hierarchies
- Evolution of Cooperation
Fairness in Primates
- Female-Female Competition
Fostering Values of Fairness
- Hunter-Gatherer Societies

- Intertribal Warfare

- Male-Male Competition

- Peer Groups

- Peer Relationships in Childhood

- Primate Cooperation

- Resource Competition

- Social Hierarchies

- Status Competition

\section{References}

Benenson, J. F., Markovits, H., Hultgren, B., Nguyen, T., Bullock, G., \& Wrangham, R. (2013). Social exclusion: More important to human females than males. PLoS One, 8(2), e55851. https://doi.org/10.1371/journal. pone. 0055851 .

Blake, P. R., Corbit, J., Callaghan, T. C., \& Warneken, F. (2016). Give as I give: Adult influence on children's giving in two cultures. Journal of Experimental Child Psychology, 152, 149-160. https://doi.org/10.1016/j. jecp.2016.07.010.

Boehm, C., Barclay, H. B., Dentan, R. K., Dupre, M.-C., Hill, J. D., Kent, S., Knauft, B. M., Otterbein, K. F., \& Rayner, S. (1993). Egalitarian behavior and reverse dominance hierarchy [and comments and reply]. Current Anthropology, 34(3), 227-254. https://doi.org/ 10.1086/204166.

Boyd, R., \& Richerson, P. J. (1992). Punishment Allows the Evolution of Cooperation (or Anything Else) in Sizable Groups. Ethology and Sociobiology, 13(3), 171-195. https://doi.org/10.1016/0162-3095(92) 90032-Y.

Brosnan, S. F., \& de Waal, F. B. M. (2003). Monkeys reject unequal pay. Nature, 425(6955), 297-299. https://doi. org/10.1038/Nature01963.

Calcott, B. (2008). The other cooperation problem: Generating benefit. Biology \& Philosophy, 23(2), 179-203. https://doi.org/10.1007/s10539-007-9095-5.

Call, J. (2009). Contrasting the social cognition of humans and nonhuman apes: The shared intentionality hypothesis. Topics in Cognitive Science, 1(2), 368-379.

Callaghan, T., \& Corbit, J. (2018). Early prosocial development across cultures. Current Opinion in Psychology, 20, 102-106. https://doi.org/10.1016/j. copsyc.2017.07.039.

Clegg, J. M., \& Legare, C. H. (2016). A cross-cultural comparison of children's imitative flexibility. Developmental Psychology, 52(9), 1435-1444. https://doi.org/ 10.1037/dev0000131.

Engelmann, J. M., Herrmann, E., \& Tomasello, M. (2012). Five-year olds, but not chimpanzees, attempt to manage their reputations. PLoS One, 7(10), e48433. https://doi. org/10.1371/journal.pone.0048433.

Gergely, G., \& Csibra, G. (2006). Sylvia's recipe: The role of imitation, and pedagogy in the transmission of cultural knowledge. In N. J. Enfield \& S. C. Levinson 
(Eds.), Roots of human sociality: Culture, cognition and interaction (pp. 229-255). New York: Berg Press.

Gonzalez-Cabrera, I. (2017). On social tolerance and the evolution of human normative guidance. The British Journal for the Philosophy of Science. https://aca demic.oup.com/bjps/advance-article-abstract/doi/10. 1093/bjps/axx017/4345800.

Henrich, J. P., Boyd, R., Bowles, S., Camerer, C., Fehr, E., Gintis, H., Mc Elreath, R., Alvard, M., Barr, A., Ensminger, J., Henrich, N. S., Hill, K., Gil-White, F., Gurven, M., Marlowe, F. W., Patton, J. Q., \& Tracer, D. (2005). "Economic man" in cross-cultural perspective: Behavioral experiments in 15 small-scale societies. Behavioral and Brain Sciences, 28(06), 795-815. https://doi.org/10.1017/S0140525X05000142.

Hrdy, S. B. (2009). Mothers and others: The evolutionary origins of mutual understanding. Cambridge, MA: Belknap Press of Harvard University Press.

Kramer, K. L. (2010). Cooperative breeding and its significance to the demographic success of humans. Annual Review of Anthropology, 39(1), 417-436. https://doi. org/10.1146/annurev.anthro.012809.105054.

Liszkowski, U., Carpenter, M., Striano, T., \& Tomasello, M. (2006). 12- and 18-month-olds point to provide information for others. Journal of Cognition and Development, 7(2), 173-187. https://doi.org/10.1207/ s15327647jcd0702_2.

McAuliffe, K., Blake, P. R., Kim, G., Wrangham, R. W., \& Warneken, F. (2013). Social influences on inequity aversion in children. PLoS One, 8(12), e80966. https://doi.org/10.1371/journal.pone.0080966.

McAuliffe, K., Blake, P. R., Steinbeis, N., \& Warneken, F. (2017). The developmental foundations of human fairness. Nature Human Behaviour, 1(2), 0042. https://doi.org/10.1038/s41562-016-0042.

Norenzayan, A. (2013). Big gods: How religion transformed cooperation and conflict. Princeton: Princeton University.

Rakoczy, H., \& Schmidt, M. F. H. (2013). The early ontogeny of social norms. Child Development Perspectives, 7(1), 17-21. https://doi.org/10.1111/Cdep.12010.

Rogers, D. S., Deshpande, O., \& Feldman, M. W. (2011). The spread of inequality. PLoS One, 6(9), e24683. https://doi.org/10.1371/journal.pone.0024683.
Salali, G. D., Juda, M., \& Henrich, J. (2015). Transmission and development of costly punishment in children. Evolution and Human Behavior, 36(2), 86-94. https:// doi.org/10.1016/j.evolhumbehav.2014.09.004.

Sandel, A. A., Reddy, R. B., \& Mitani, J. C. (2017). Adolescent male chimpanzees do not form a dominance hierarchy with their peers. Primates, 58(1), 39-49. https://doi.org/10.1007/s10329-016-0553-z.

Sheskin, M., Ashayeri, K., Skerry, A., \& Santos, L. R. (2014). Capuchin monkeys (Cebus apella) fail to show inequality aversion in a no-cost situation. Evolution and Human Behavior, 35(2), 80-88. https://doi. org/10.1016/j.evolhumbehav.2013.10.004.

Smith, D., Schlaepfer, P., Major, K., Dyble, M., Page, A. E., Thompson, J., Chaudhary, N., Salali, G. D., Mace, R., Astete, L., Ngales, M., Vinicius, L., \& Migliano, A. B. (2017). Cooperation and the evolution of hunter-gatherer storytelling. Nature Communications, 8(1), 1853. https://doi.org/10.1038/s41467-017-02036-8.

Thompson, J. L., \& Nelson, A. J. (2011). Middle childhood and modern human origins. Human Nature, 22(3), 249-280. https://doi.org/10.1007/s12110-011-9119-3.

Tomasello, M., \& Carpenter, M. (2007). Shared intentionality. Developmental Science, 10(1), 121-125. https:// doi.org/10.1111/j.1467-7687.2007.00573.x.

Tomasello, M., \& Gonzalez-Cabrera, I. (2017). The role of ontogeny in the evolution of human cooperation. Human Nature, 28(3), 274-288. https://doi.org/ 10.1007/s12110-017-9291-1.

Warneken, F. (2018). How children solve the two challenges of cooperation. Annual Review of Psychology, 69(1), 205-229. https://doi.org/10.1146/annurevpsych-122216-011813.

Warneken, F., Chen, F., \& Tomasello, M. (2006). Cooperative activities in young children and chimpanzees. Child Development, 77(3), 640-663. https://doi.org/ 10.1111/j.1467-8624.2006.00895.x.

Watts, J., Sheehan, O., Atkinson, Q. D., Bulbulia, J., \& Gray, R. D. (2016). Ritual human sacrifice promoted and sustained the evolution of stratified societies. Nature, 532(7598), 228-231. https://doi.org/10.1038/ nature 17159 . 